\title{
Delayed Mesolimbic System Alteration in a Developmental Animal Model of Schizophrenia
}

\author{
Yukiori Goto and Patricio O'Donnell \\ Center for Neuropharmacology and Neuroscience, Albany Medical College, Albany, New York 12208
}

Pharmacological and imaging studies indicate that the prefrontal cortex and nucleus accumbens and their dopamine innervation are central elements of the pathophysiology of schizophrenia. Although symptoms typically appear in young adults, a developmental component has been suggested, primarily in the hippocampus. A neonatal hippocampal lesion in rats and monkeys produces changes resembling schizophrenia symptoms only after the animals reach adulthood, indicating that this procedure could be used as a developmental animal model of this disorder. Here, we explored whether the dopamine projection to the nucleus accumbens becomes functionally altered in these animals. In vivo intracellular recordings revealed abnormal responses in accumbens neurons to activation of their dopamine afferents in adult but not prepubertal animals with a neonatal lesion. This alteration was absent after antipsychotic drug treatment. These results indicate that neonatal hippocampal damage can result in delayed functional deficits in the mesolimbic system, providing a link between the developmental hippocampal deficit and altered dopamine systems postulated to occur in schizophrenia.

Key words: nucleus accumbens; schizophrenia; animal model; hippocampus; dopamine; electrophysiology; antipsychotic
A neonatal lesion of the ventral hippocampus $(\mathrm{VH})$ results in behavioral alterations in adult rodents (Lipska et al., 1993) and monkeys (Bachevalier et al., 1999a). These include exaggerated responses to stress, dopamine (DA) agonists, and NMDA antagonists, which become apparent only after the animals reach adulthood (Lipska et al., 1993; Al-Amin et al., 2000). In addition, a neonatal VH lesion reduces social interactions (Sams Dodd et al., 1997; Bachevalier et al., 1999a) and causes cognitive deficits, including altered sensorimotor gating (Lipska et al., 1996) and working memory (Chambers et al., 1996; Bachevalier et al., 1999b). These findings provided apparent validity for this manipulation as an animal model of schizophrenia, because the delayed behavioral manifestations resemble phenomena observed in this disease (Lipska and Weinberger, 2000).

A neonatal $\mathrm{VH}$ lesion also produces cytoarchitechtonical alterations resembling postmortem findings in schizophrenia patients in brain regions targeted by $\mathrm{VH}$ projections. For example, a decrease of interneurons in the prefrontal cortex (PFC), measured as cells expressing the GABA synthesis enzyme GAD67, has been reported in neonatal VH-lesioned animals (Lipska and Weinberger, 2000) and in patients (Volk et al., 2000). Thus, this lesion may produce developmental alterations mimicking those present in schizophrenia (Waddington, 1993; Weinberger and Lipska, 1995), a disorder in which structural hippocampal abnor-

\footnotetext{
Received June 7, 2002; revised July 26, 2002; accepted Aug. 1, 2002.

This work was supported by United States Public Health Service Grants MH57683 and MH-60131 and by a National Alliance for Research on Schizophrenia and Depression Independent Investigator Award to P.O. P.O. is a Wodecroft investigator. We thank Barbara L. Lewis for her excellent technical assistance, Drs. Barbara K. Lipska and Daniel R. Weinberger for teaching us the neonatal hippocampal lesion method, and Brian Lowry (University of Pittsburgh, Pittsburgh, PA) for developing and providing the software used for data acquisition and analysis (Neuroscope).

Correspondence should be addressed to Dr. Patricio O'Donnell, Albany Medical College (MC-136), Center for Neuropharmacology and Neuroscience, Albany, NY 12208. E-mail: odonnep@mail.amc.edu.

Copyright (ㄷ) 2002 Society for Neuroscience $0270-6474 / 02 / 229070-08 \$ 15.00 / 0$
}

malities (Kovelman and Scheibel, 1984; Weinberger, 1999) suggest altered neuronal migration.

Determination of the construct validity of this model is of great importance to our understanding of this devastating disease. Important elements to assess in this regard are functional deficits arising from this procedure and their response to antipsychotic medication. We have shown recently that the response of PFC pyramidal neurons to stimulation of dopaminergic afferents that originated in the ventral tegmental area (VTA) is altered in animals with a neonatal VH lesion (O'Donnell et al., 2002). A critical missing link is the possibility of subcortical functional alterations arising from a neonatal VH lesion. The nucleus accumbens (NAcc) is an important component of schizophrenia pathophysiology (O'Donnell and Grace, 1998). Current treatment strategies in schizophrenia target the NAcc DA innervation (Grace, 1992). In addition, the NAcc receives convergent information from other areas affected in schizophrenia, such as the PFC, hippocampus, and amygdala (O'Donnell and Grace, 1995). The NAcc receives an important projection from the VH (Groenewegen et al., 1987), and NAcc neuron electrical activity is dependent primarily on inputs from this region (O'Donnell and Grace, 1995; Goto and O'Donnell, 2001a). It is thought that DA systems may become altered as a consequence of cortical deficits in schizophrenia (O'Donnell and Grace, 1998). This alteration, causing improper flow of information through the NAcc, would in turn impair PFC function (O'Donnell et al., 1999). In this study, we tested whether the response of NAcc neurons to DA afferents is affected in adult rats with a neonatal $\mathrm{VH}$ lesion to establish a link between a hippocampal developmental deficit and subcortical DA function. To assess the developmental nature of the changes observed, the results were compared with sham and neonatally lesioned animals tested before puberty and with animals that received a lesion similar to those in adults.

\section{MATERIALS AND METHODS}

Animals. Pregnant Sprague Dawley rats at $11-15$ d of gestation were obtained from Taconic Farms (Germantown, NY). For adult lesions, 
male Sprague Dawley rats weighing 225-250 gm were used. All experimental procedures were performed according to the United States Public Health Service Guide for the Care and Use of Laboratory Animals and approved by the Albany Medical College Institutional Animal Care and Use Committee.

Surgery. Neonatal VH lesions were performed in male pups at postnatal day 6 (P6) to P8. Four to nine pups were used for every surgery; approximately one-half of the pups were lesioned, and a sham operation was performed in the other half. Bilateral $\mathrm{VH}$ lesions were performed as described previously (Lipska et al., 1993). Briefly, pups were anesthetized by placing them in ice for 10-15 min. The pups were then placed on a stereotaxic apparatus (David Kopf Instruments, Tujunga, CA), and incisions were made in the skin. For a neonatal $\mathrm{VH}$ lesion, $0.3 \mu \mathrm{l}$ of ibotenic acid (10 $\mu \mathrm{g} / \mu \mathrm{l}$; Sigma, St Louis, MO) in $0.1 \mathrm{M}$ PBS, pH 7.4, was delivered bilaterally to the $\mathrm{VH}$ (anteroposterior to bregma, $-3.0 \mathrm{~mm}$; lateral, $\pm 3.5 \mathrm{~mm}$; ventral, $-5.0 \mathrm{~mm}$ ) at a speed of $0.15 \mu \mathrm{l} / \mathrm{min}$. Shamoperated animals received the same amount of artificial CSF (aCSF). After finishing the injection, the needle was kept in place for 3 additional minutes. The incision was closed with clips, and the pups were warmed with heating pads $\left(\sim 37^{\circ} \mathrm{C}\right)$ until body temperature had completely recovered.

In one control group, a similar $\mathrm{VH}$ lesion was performed in adult rats (older than P56). Rats were initially anesthetized with Equithesin (40 $\mathrm{ml} / \mathrm{kg}$, i.p.) and placed on the stereotaxic frame. Bilateral injections of either ibotenic acid for lesion or aCSF for sham were performed into the hippocampi (4.4 $\mathrm{mm}$ caudal to bregma; $\pm 5.0 \mathrm{~mm}$ lateral from midline; 6.0 and $8.0 \mathrm{~mm}$ ventral from brain surface: $0.3 \mu \mathrm{l}$ at each site, for a total of $0.6 \mu \mathrm{l}$ per side). Adult-lesioned rats were allowed $\geq 2$ weeks for recovery before recordings were conducted.

Recordings. For recording sessions, the animals were anesthetized with chloral hydrate $(400 \mathrm{mg} / \mathrm{kg}$, i.p.) and placed on the stereotaxic apparatus. Burr holes were drilled in the skull to allow for the placement of recording and stimulating electrodes. Intracellular electrodes were made from $1 \mathrm{~mm}$ outer diameter Omegadot borosilicate glass tubing (World Precision Instruments, Sarasota, FL) pulled with a P-97 Flaming-Brown puller (Sutter Instruments, Novato, CA). Electrodes were filled with $2 \mathrm{M}$ potassium acetate and 2\% neurobiotin and had a resistance of 42-99 $\mathrm{M} \Omega$. Intracellular electrodes were lowered into the NAcc $(1.4-2.1 \mathrm{~mm}$ rostral to bregma; $1.0-2.0 \mathrm{~mm}$ lateral from midline; $5.8-8.4 \mathrm{~mm}$ ventral from brain surface). Recording electrodes were advanced with a hydraulic manipulator (Trent Wells), and their activity was monitored on a Philips PM3337 oscilloscope (Fluke Corp.). Intracellular signals were amplified using an IR-283 Neurodata amplifier (Cygnus Technology), filtered at $0.3-3 \mathrm{kHz}$ with an eight pole Bessel filter (FLA-01; Cygnus Technology), digitized with an interface board (DAP3215a; Microstar Laboratories, Bellevue, WA) at $10 \mathrm{kHz}$, and fed to a computer (Gateway PII 266) for off-line analyses. Once a stable impalement was obtained, baseline recordings were performed. Only neurons showing at least -50 $\mathrm{mV}$ resting membrane potential with overshooting spikes were analyzed and included in the study. All data handling was performed using custom-made software (Neuroscope).

Electrical stimulation. Concentric bipolar electrodes with $0.5 \mathrm{~mm}$ between tips (NE-100X; Rhodes Instruments) were used for electrical stimulation. The electrodes were placed in the VTA $(5.8 \mathrm{~mm}$ caudal from bregma; $0.5 \mathrm{~mm}$ lateral from midline; $8.4 \mathrm{~mm}$ ventral from brain surface) and medial PFC (3.5 mm rostral from bregma; $0.5 \mathrm{~mm}$ lateral from midline; $4.0 \mathrm{~mm}$ ventral from brain surface) ipsilaterally to the NAcc. Current pulses were generated by stimulus isolation units driven by a Master 8 Stimulator (AMPI, Jerusalem, Israel), and stimulation protocols were controlled by the computer. VTA electrical stimulation was performed by delivering five $0.5 \mathrm{msec}, 1.0 \mathrm{~mA}$ current pulses at $20 \mathrm{~Hz}$ every $10 \mathrm{sec}$ to mimic DA cell burst firing. Single pulses of the same current intensity were applied to the PFC.

Drug treatment. Haloperidol (Haldol; $5 \mathrm{mg} / \mathrm{ml}$ in methylparaben, propylparaben, and lactic acid, pH 3.0-3.6; McNeil Pharmaceutical, Spring House, PA) was dissolved in drinking water. Water intake was calculated daily, and drug concentration was adjusted daily to approximate 1.0 $\mathrm{mg} \cdot \mathrm{kg}^{-1} \cdot \mathrm{d}^{-1}$, a dose known to induce changes in the striatal regions (Caboche et al., 1992; Marcus et al., 1997) and the minimal dose that affects evoked DA overflow in the NAcc (Feasey-Truger et al., 1995). Drug administration started at P56 and continued for $\geq 3$ weeks (21-27 d) until the day before the recording session. The same amount of water without drug was given to control animals for $\sim 3$ weeks.

Histology. After completion of the experiments, recording sites were marked with neurobiotin ejected from intracellular electrodes by passing
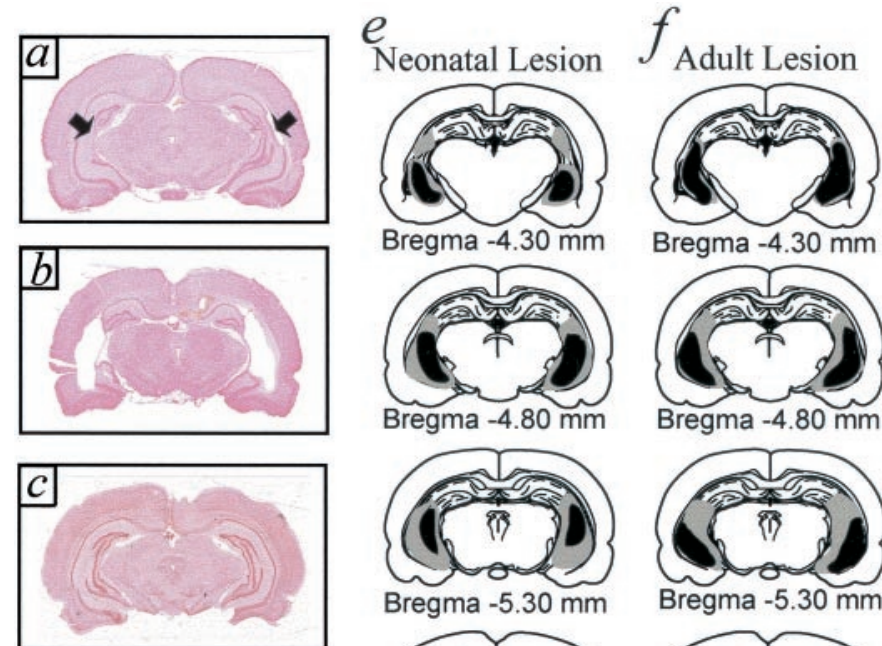

Bregma $-5.30 \mathrm{~mm}$
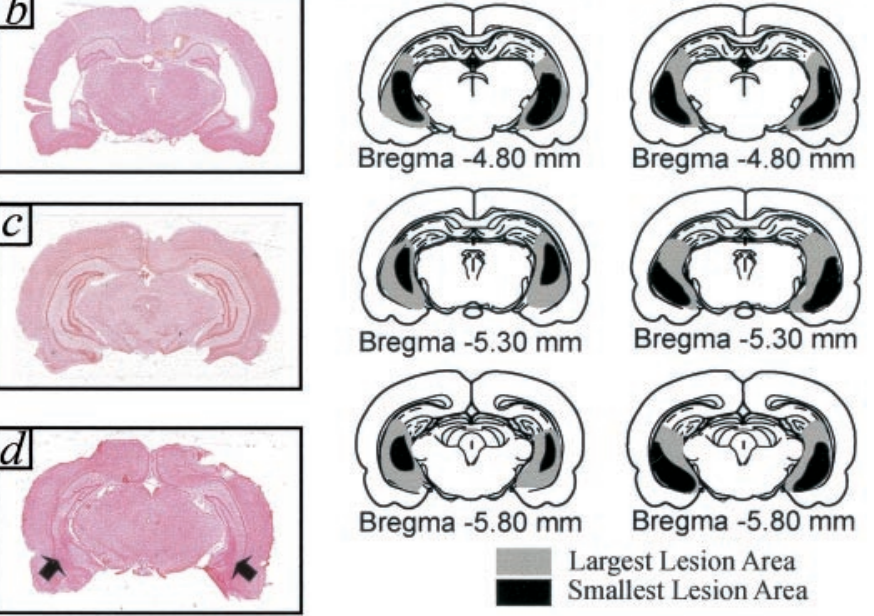

Bregma $-5.30 \mathrm{~mm}$

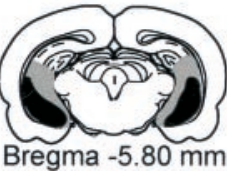

Largest Lesion Area

Smallest Lesion Area

Figure 1. Histology of VH lesions. $a$, Small neonatal VH lesions resulted in cell loss and structural alteration in the remaining hippocampus (arrows). $b$, One of the largest neonatal $\mathrm{VH}$ lesions showing profound cell loss in the $\mathrm{VH}$ and enlarged ventricles. $c$, Brain section of a neonatal sham rat at the same level as in $b$; no hippocampal loss is observed. $d$, Brain section of an adult $\mathrm{VH}$ lesion. $e$, Schematic diagrams illustrating neonatal VH lesions. Gray and black areas show the largest and smallest extent of $\mathrm{VH}$ lesions, respectively. $f$, Schematic diagrams showing extent of adult VH lesions.

positive current $(1.0 \mathrm{nA}, 200 \mathrm{msec}$ pulses at $2 \mathrm{~Hz})$ for $\geq 5 \mathrm{~min}$. Animals were given a lethal dose of pentobarbital $(100 \mathrm{mg} / \mathrm{kg})$ and perfused transcardially with ice-cold saline followed by $4 \%$ paraformaldehyde. Brains were removed from the skull, cryoprotected in $30 \%$ sucrose, and sectioned using a freezing microtome. Serial sections $50 \mu \mathrm{m}$ thick were cut coronally. Neurobiotin-injected sections were incubated further in $0.4 \%$ Triton X-100 (Sigma) in PBS for 1-2 hr, followed by $2 \mathrm{hr}$ in Vectastain Elite ABC reagent (Vector Laboratories, Burlingame, CA). After a series of rinses, sections were reacted with 3,3'-diaminobenzidine and urea-hydrogen peroxide (Sigma FAST DAB set). All sections were mounted on gelatin-coated slides, air-dried for $24 \mathrm{hr}$, cleared in xylene, coverslipped in Permount, and examined on an Olympus CH30 microscope (Olympus Optical, Tokyo, Japan). The locations of intracellularly recorded neurons were identified according to the atlas of Paxinos and Watson (1998).

\section{RESULTS}

\section{Lesion}

Bilateral damage of the $\mathrm{VH}$ was observed in rats in which ibotenic acid was injected at P6-P8. Lesions included the ventral part of CA3, CA1, dentate gyrus, and subiculum; the dorsal hippocampus was spared in all cases. The neonatal VH lesion was characterized by cell loss, pyramidal cell disorientation in the remaining hippocampus, and enlargement of ventricles (Fig. 1a,b,e). Sham animals did not show any obvious alteration of hippocampal architecture (Fig. 1c). Adult VH lesions resulted in an extent of $\mathrm{VH}$ loss similar to that observed with neonatal VH lesions (Fig. $1 d, f)$. In all groups, lesion sizes were variable. Animals with small lesions (Fig. 1a) and large lesions (Fig. 1b) exhibited similar electrophysiological properties. Therefore, the data were pooled regardless of lesion size. 


\section{Membrane potential states in NAcc neurons from lesioned rats}

Fourteen cells were recorded from 10 adult rats (older than P56) that had received a neonatal $\mathrm{VH}$ lesion, and eight cells were recorded from six adult rats with a neonatal sham operation. As reported previously in normal animals (O'Donnell and Grace, 1995; Goto and O’Donnell, 2001a), most NAcc neurons exhibited membrane potential fluctuations characterized by a very negative resting membrane potential (down state) interrupted by plateau depolarizations (up state). The presence of such membrane potential states was ascertained by fitting membrane potential histograms to dual Gaussian functions (Fig. 2). Bimodal membrane potential distributions were observed in $71 \%(n=10$ of 14$)$ and $75 \%$ ( $n=6$ of 8 ) of neurons in neonatally lesioned and sham rats, respectively. Thus, a neonatal $\mathrm{VH}$ lesion does not result in a decrease in the proportion of neurons with up/down membrane potential fluctuations.

Similar recordings were performed in prepubertal rats $(\mathrm{P} 28-$ $\mathrm{P} 35$ ) with a neonatal VH lesion or sham operation. Both groups showed a similar proportion of neurons with membrane potential fluctuations ( $n=6$ of 8 neurons in eight rats, $75 \%$ in neonatal $\mathrm{VH}$ lesion; $n=4$ of 6 cells in seven rats, $67 \%$ in neonatal sham). The down state of NAcc neurons from these younger animals was more depolarized than in adults (one-way ANOVA; $F_{(5,37)}=$ 3.95; $p<0.01$ ) (Table 1; Fig. $2 b$ ). The amplitude of membrane fluctuations in $\mathrm{P} 28-\mathrm{P} 35$ groups was also smaller than in adult groups $\left(F_{(4,26)}=2.97 ; p<0.05\right)$ (Fig. $\left.2 b\right)$. Membrane potential distribution histograms of neurons recorded from P28-P35 rats could still be fitted to dual Gaussian functions (Fig. $2 b$ ), indicating the presence of up states with values close to those of the down state. This is probably the consequence of the less polarized down state in these immature animals.

Recordings were also performed from rats with a similar lesion performed at adult ages (nine lesioned and seven sham rats). In adult-lesioned rats, up transitions were observed in only 1 of 10 neurons, a proportion significantly lower than in rats with neonatal lesion and adult-sham operation (Table 1). Therefore, most membrane potential distribution histograms in this group could not be fitted to a dual Gaussian function. Instead, they were fitted to single normal functions, indicating absence of the up state (Fig. $2 c$ ). Small spontaneous EPSPs were observed in adult VHlesioned rats (Fig. $2 c$, inset). In adult sham rats, up transitions were readily observed ( $n=7$ of $11 ; 64 \%$ ) (Table 1$)$. Thus, unlike neonatal VH lesions, adult $\mathrm{VH}$ lesions eliminated membrane potential fluctuations in the NAcc.

\section{Increased regularity of spontaneous membrane potential fluctuations in rats with a neonatal ventral hippocampal lesion}

The patterns of membrane potential fluctuations in adult rats that received a neonatal $\mathrm{VH}$ lesion or a sham operation were investigated. No differences in up state duration were found among groups (Table 1). The frequency domains of membrane potential fluctuations were assessed with spectral density analyses of $20 \mathrm{sec}$ epochs of recording using fast Fourier transform. In all cases, spectral density peaks were observed at $\sim 1 \mathrm{~Hz}$ (Fig. $3 a, b$ ), which corresponded to the frequency of up transitions. To analyze the regularity of up transitions, the "sequential interval state space" method (Eagan and Partridge, 1989) was used. This is essentially a three-dimensional plot of intervals between onsets of three consecutive up states. More clustered sequential up transition time points were found in adult rats with a neonatal $\mathrm{VH}$ lesion
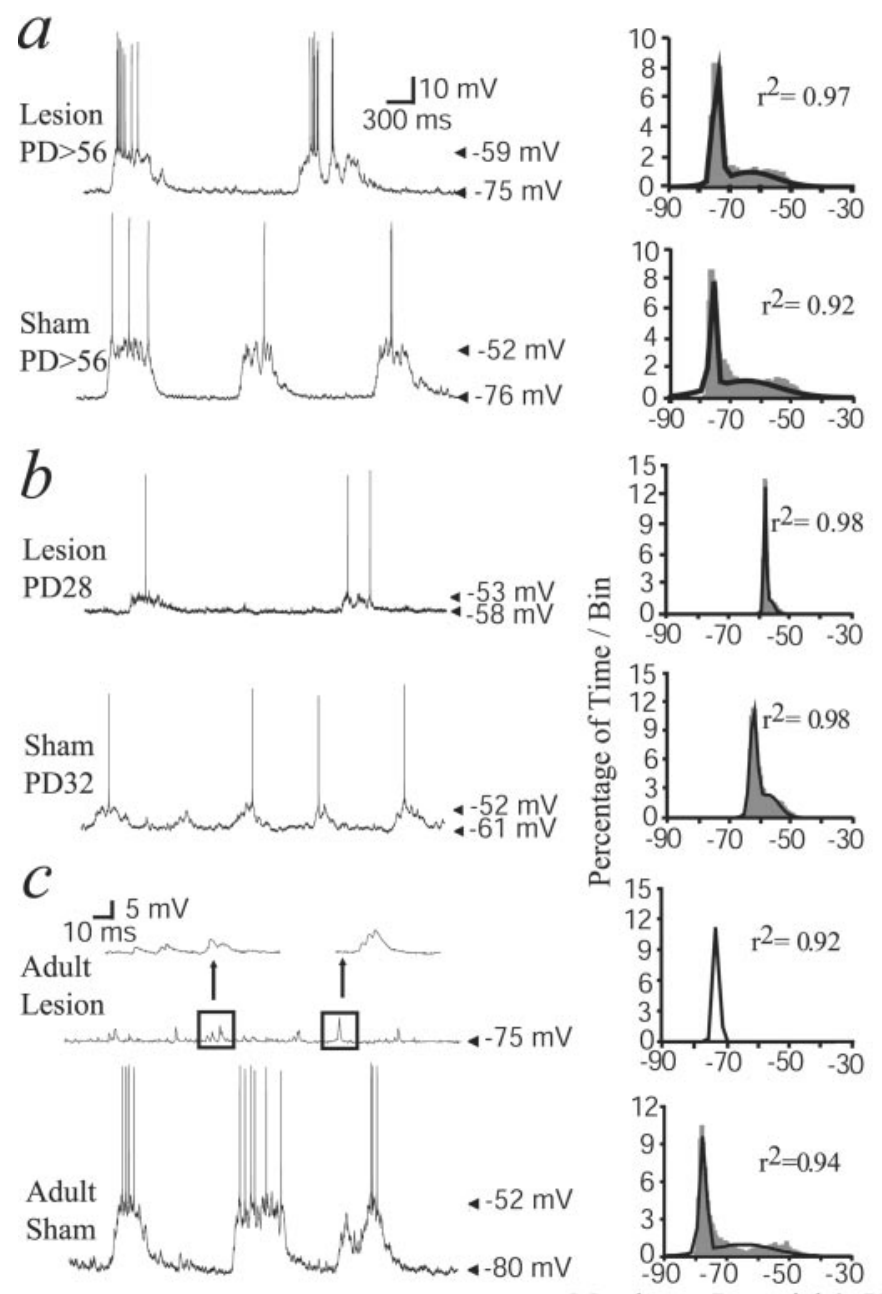

Membrane Potential ( $\mathrm{mV})$

Figure 2. Spontaneous membrane potential fluctuations in NAcc neurons from VH-lesioned and sham rats. $a$, Representative tracing of spontaneous NAcc neuron activity recorded from a neonatally lesioned (top) and a sham (bottom) rat at P56 or older (PD56) showing typical spontaneous membrane potential fluctuations. The histograms on the right illustrate membrane potential distributions from the recordings shown on the left. Dark lines are the dual Gaussian functions that best fit to the distribution. $b$, Recordings and histograms obtained from neonatally lesioned and sham rats recorded at P28-P35. In these rats, spontaneous membrane potential fluctuations were also observed, although amplitude of up transitions was small and the down state was more depolarized. $c$, Recordings from adult-lesioned and sham rats showing that up transitions were absent in VH-lesioned but not in sham rats. Insets, Faster time scale of a trace from an adult-lesioned rat showing spontaneous EPSP-like depolarizations that did not match the criteria of $\geq 100$ msec duration used for up states. The membrane potential distribution histogram in neurons recorded from adult-lesioned rats did not fit to a dual but rather to a single Gaussian function.

than in adult rats with a neonatal sham operation (Fig. 3c). This suggests that up transitions were more regular in neonatally lesioned animals. In addition, the coefficient of variation $(\mathrm{CV})$ for intervals between up transitions was significantly lower in adult rats with a neonatal lesion than in neonatal sham rats $(0.30 \pm 0.18$ and $0.56 \pm 0.08$, respectively; $p<0.05$; unpaired $t$ test) (Fig. $3 e$ ). The CV of up transitions in rats with a neonatal $\mathrm{VH}$ lesion tested at P28-P35 was also significantly lower than in rats with a neonatal sham operation tested at the same age $(0.40 \pm 0.11$ in neonatal VH lesion; $0.60 \pm 0.04$ in neonatal sham; $p<0.05$; 
Table 1. In vivo electrophysiological properties of NAcc neurons

\begin{tabular}{|c|c|c|c|c|c|c|c|c|}
\hline & \multicolumn{2}{|l|}{$>$ P56 } & \multicolumn{2}{|l|}{$\mathrm{P} 28-\mathrm{P} 35$} & \multicolumn{2}{|l|}{ Adult lesion } & \multirow{2}{*}{$\begin{array}{l}\text { Haloperidol, } \\
\text { neonatal } \\
\text { VH lesion }\end{array}$} & \multirow{2}{*}{$\begin{array}{l}\text { Vehicle, neo- } \\
\text { natal VH le- } \\
\text { sion }\end{array}$} \\
\hline & $\begin{array}{l}\text { Neonatal } \\
\text { VH lesion }\end{array}$ & $\begin{array}{l}\text { Neonatal } \\
\text { sham }\end{array}$ & $\begin{array}{l}\text { Neonatal VH } \\
\text { lesion }\end{array}$ & $\begin{array}{l}\text { Neonatal } \\
\text { sham }\end{array}$ & $\begin{array}{l}\text { Adult } \\
\text { VH lesion }\end{array}$ & Adult sham & & \\
\hline $\begin{array}{l}\text { Number of up/down cel } \\
\text { total recorded }\end{array}$ & $10 / 14$ & $6 / 8$ & $6 / 8$ & $4 / 6$ & $1 / 10$ & $7 / 11$ & $27 / 35$ & $7 / 7$ \\
\hline Percentage & $71 \%$ & $75 \%$ & $75 \%$ & $67 \%$ & $10 \%{ }^{a}$ & $64 \%$ & $77 \%$ & $100 \%$ \\
\hline \multicolumn{9}{|c|}{ Membrane potential (mV) } \\
\hline Down & $-74.1 \pm 12.0$ & $-78.8 \pm 9.0$ & $-65.1 \pm 6.9$ & $-62.8 \pm 5.7$ & $-76.0 \pm 7.4$ & $-75.1 \pm 10$ & $-77.5 \pm 7.3$ & $-78.1 \pm 5.5$ \\
\hline Up & $-67.3 \pm 11.3$ & $-60.8 \pm 12.9$ & $-56.9 \pm 4.2$ & $-56.3 \pm 8.0$ & - & $-65.0 \pm 6.6$ & $-65.3 \pm 8.8$ & $-67.7 \pm 8.1$ \\
\hline Input resistance $(\mathrm{M} \Omega)$ & $43.4 \pm 13.4$ & $37.3 \pm 16.1$ & $27.8 \pm 14.2$ & $44.5 \pm 11.7$ & $38.4 \pm 18.3$ & $48.6 \pm 16.5$ & $46.6 \pm 20.1$ & $48.4 \pm 12.4$ \\
\hline Time constant (msec) & $6.0 \pm 1.9$ & $6.5 \pm 2.4$ & $5.0 \pm 1.7$ & $6.5 \pm 2.3$ & $4.9 \pm 0.6$ & $6.7 \pm 1.3$ & $6.5 \pm 2.3$ & $6.8 \pm 3.5$ \\
\hline Up frequency $(\mathrm{Hz})$ & $0.49 \pm 0.21$ & $0.81 \pm 0.13$ & $0.67 \pm 0.37$ & $0.59 \pm 0.10$ & - & $0.64 \pm 0.31$ & $0.49 \pm 0.21$ & $0.81 \pm 0.13$ \\
\hline Up duration (msec) & $357 \pm 182$ & $605 \pm 199$ & $210 \pm 134$ & $168 \pm 136$ & - & $359 \pm 239$ & $510 \pm 310$ & $287 \pm 141$ \\
\hline Firing rate $(\mathrm{Hz})$ & $\begin{array}{l}1.46 \pm 0.84 \\
(n=4 / 14)^{b}\end{array}$ & $\begin{array}{c}0.51 \pm 0 \\
(n=1 / 8)\end{array}$ & $\begin{array}{l}1.74 \pm 2.07 \\
(n=3 / 8)\end{array}$ & $\begin{array}{l}1.58 \pm 0.87 \\
(n=3 / 6)^{c}\end{array}$ & - & $\begin{array}{l}2.61 \pm 2.08 \\
(n=3 / 11)\end{array}$ & $\begin{array}{l}2.12 \pm 2.27 \\
(n=8 / 35)\end{array}$ & $\begin{array}{r}0 \pm 0 \\
(n=0 / 7)\end{array}$ \\
\hline
\end{tabular}

All values are mean $\pm \mathrm{SD}$.

${ }^{a}$ Fisher's exact test compared with neonatal VH lesion at $>$ P56 $(p=0.0042)$; compared with adult sham $(p=0.017)$.

${ }^{b}$ Number of cells that showed spontaneous spike firing.

${ }^{c}$ Unpaired $t$ test compared with vehicle $(p<0.00001)$.

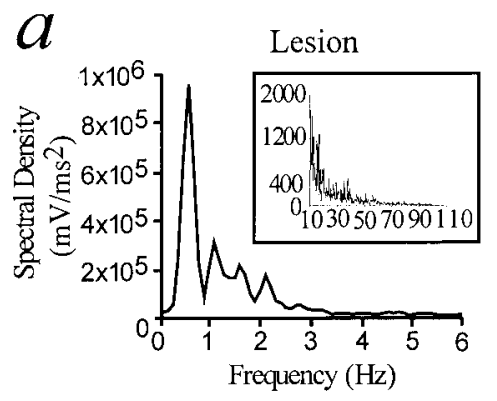

$b$

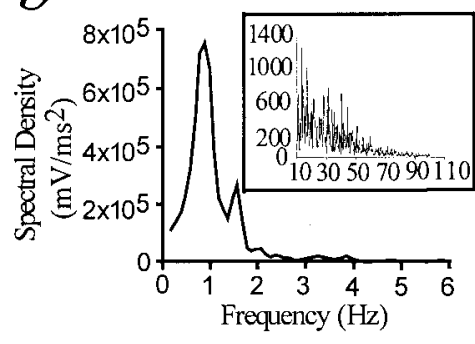

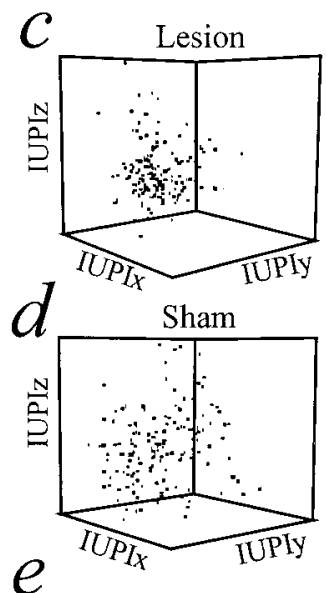

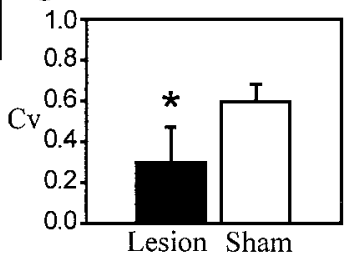

Figure 3. Analyses of spontaneous membrane potential fluctuations in neonatally lesioned and sham rats. All examples shown here were obtained from the recordings shown in Figure $2 a$. $a$, Power spectral density from a representative neuron recorded from a neonatally lesioned rat (0-6 Hz; inset shows higher frequency ranges), in which a peak is observed close to $1 \mathrm{~Hz}$. $b$, Similar power spectral density analysis from a neuron recorded in a rat with a neonatal sham operation. $c$, Sequential interval analysis in neonatally lesioned rats. IUPI, Inter-up interval. IUPIx, IUPIy, and IUPIz refer to consecutive intervals between up state onsets. $d$, Similar sequential up interval analysis in a neonatal sham rat. More scattered points are observed than in $c$, indicating a less regular time series. $e$, Comparison of the $\mathrm{CV}$ of up state intervals showing that variability is significantly decreased in neonatally lesioned rats compared with neonatal sham rats. ${ }^{*} p<0.05$ (unpaired $t$ test).

unpaired $t$ test). These results indicate that a neonatal $\mathrm{VH}$ lesion yields more regular up transitions, an effect that can be observed in both prepubertal and postpubertal animals and in animals with both small and large lesions.

\section{Passive membrane properties of NAcc neurons}

Passive membrane properties of NAcc neurons were investigated by intracellular current injection. Neurons from all groups exhibited similar input resistance $\left(F_{(5,27)}=1.06 ; p>0.05\right)$ and time constant $\left(F_{(5,27)}=1.05 ; p>0.05\right)$ (Table 1$)$. Inward rectification was observed in response to positive current injection in all treatment groups. Thus, membrane properties were not affected by any of the lesions performed in this study.

\section{Altered response to VTA stimulation in adult rats with a neonatal VH lesion}

To assess the response of NAcc neurons to activation of the mesolimbic pathway, the VTA was electrically stimulated. The VTA is the source of DA projection to the NAcc (Thierry et al., 1973; Voorn et al., 1986). As reported previously in normal animals (Yim and Mogenson, 1988; Goto and O'Donnell, 2001b), stimulation with trains of five pulses at $20 \mathrm{~Hz}(1.0 \mathrm{~mA})$ that mimic DA cell burst firing depolarized NAcc neurons to a value similar to the up state. This was observed in all groups except in rats with an adult VH lesion (Fig. $4 a-c$ ). Peak amplitude and duration (measured as decay to half-maximal amplitude) of the evoked depolarization were not significantly different among groups (Fig. $4 d, e)$. Although adult lesions eliminated VTA-evoked depolarizations, neonatal lesions failed to do so. Together with the similar finding with spontaneous membrane potential fluctuations, this suggests that synaptic compensations may occur after the neonatal but not the adult lesion.

NAcc neuronal firing was altered in adult animals with a neonatal VH lesion during this VTA-evoked depolarization. In untreated animals (Goto and O'Donnell, 2001b) and all control groups, VTA-evoked depolarization was accompanied by a marked suppression of spike firing (Fig. $4 a-c, f$ ). In rats with a neonatal VH lesion tested after P56, however, high-frequency firing was observed during the VTA-evoked membrane depolarization (Fig. 4a,f). The number of cells with action potential firing during the VTA-evoked depolarization was significantly higher in rats with a neonatal $\mathrm{VH}$ lesion tested after P56 than in other control groups $\left(\chi^{2}=15.3 ; p=0.009 ; \chi^{2}\right.$ test) (Fig. $\left.4 f\right)$, an effect that was not correlated with lesion size. Such qualitative change 


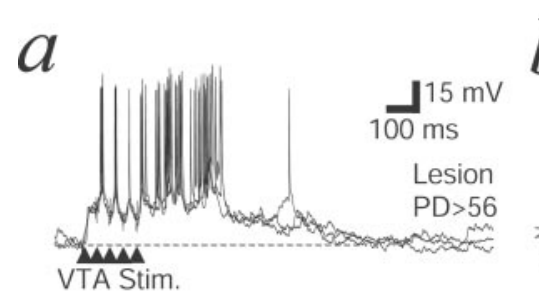

b
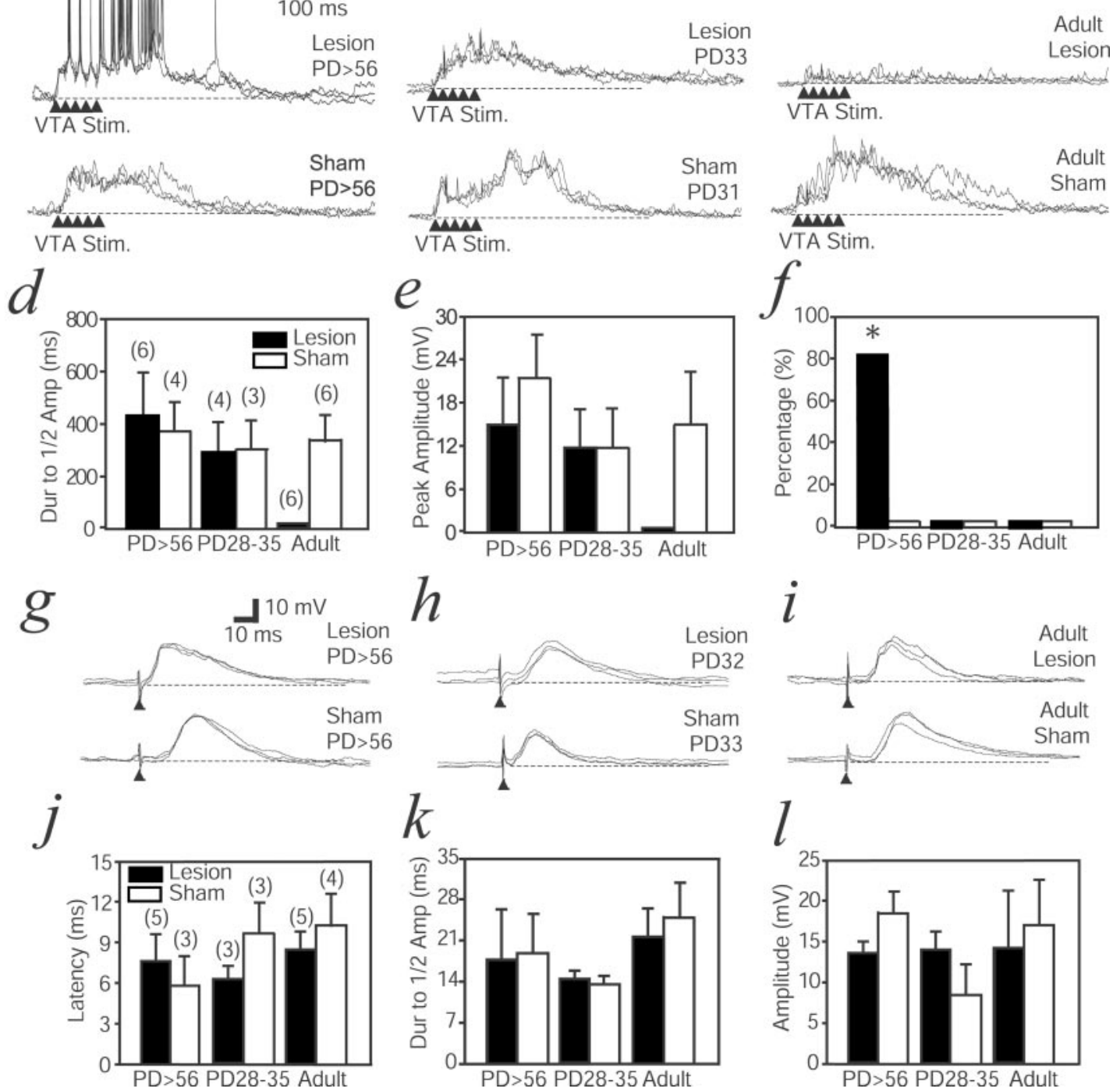

Figure 4. NAcc responses to VTA stimulation (VTA Stim.) with trains of pulses (arrowheads). a, Overlay of responses from a neonatally lesioned rat (top) and a sham rat (bottom) recorded after P56 (PD56). In the neonatally lesioned animal, high-frequency spike firing could be detected during the membrane potential depolarization. This was not observed in the sham rat. $b$, Responses in a neonatally lesioned (top) and a sham (bottom) rat recorded at P28-P35. A depolarization is observed in both cases, but no spike firing could be evoked at this early age. $c$, Responses in an adult-lesioned rat (top) and a sham rat. The adult VH lesion abolishes the VTA-evoked membrane potential depolarization. A depolarization without spike firing is observed in rats that received a sham operation as adults. $d$, Bar graph comparing duration (Dur) of evoked membrane depolarization, expressed as decay to half amplitude (Amp). Numbers in parentheses indicate the number of recordings. $e$, Bar graph comparing peak amplitude of VTA-evoked responses among all groups. $f$, Bar graph comparing the number of neurons exhibiting spike firing in response to VTA stimulation, represented as a percentage. ${ }^{*} p<0.01$ ( $\chi^{2}$ test). $g$, NAcc responses to PFC stimulation (arrowhead). An overlay of responses in a neonatally lesioned (top) and a sham (bottom) rat older than P56 is shown. EPSPs were evoked in both cases. $h$, EPSP responses in a neonatally lesioned (top) and a sham (bottom) rat at P28-P35. $i$, Similar EPSPs evoked by PFC stimulation in an adult-lesioned (top) and a sham (bottom) rat. $j, k, l$, Comparisons of PFC-evoked EPSP amplitudes, decay time to half of peak response, and latency among all groups. Numbers in parentheses indicate the number of recordings. No difference could be observed. Dashed lines in all traces indicate resting membrane potential.

in the response to activation of the mesolimbic system, absent in adult-lesioned animals, may be a consequence of developmental processes after the neonatal lesion.

\section{Intact response to PFC stimulation}

Single-pulse stimulation of the prelimbic PFC was performed to evaluate responses to PFC afferent activation. In every case, an EPSP was evoked by the stimulation (Fig. $4 g-i$ ). Neither latency, peak amplitude, nor decay of EPSP was different among groups
(Fig. $4 j-l$ ). These results indicate that a neonatal $\mathrm{VH}$ lesion did not affect NAcc responses to electrical PFC stimulation.

\section{Subchronic haloperidol treatment prevented altered responses to VTA stimulation}

In another set of animals with a neonatal VH lesion, we examined the effect of subchronic treatment with a classic antipsychotic on NAcc response to VTA stimulation. Ten rats with a neonatal VH lesion were orally treated with haloperidol dissolved in drinking 
water $\left(0.9 \pm 0.4 \mathrm{mg} \cdot \mathrm{kg}^{-1} \cdot \mathrm{d}^{-1}\right)$ for $\geq 3$ weeks starting at P56. By the end of the treatment period, all animals were catatonic, a characteristic motor side effect of neuroleptic treatment. The control group included animals with a neonatal VH lesion that received drug-free water (vehicle; $n=6$ ). Recordings were obtained from NAcc neurons in haloperidol-treated and vehicle rats. Bimodal membrane potential distributions were observed in both haloperidol-treated ( $n=27$ of 35 neurons in 10 rats; 77\%) and vehicle $(n=7$ of 7 neurons in six rats; 100\%) animals. However, the membrane potential of NAcc neurons was noisy in both up and down states in haloperidol-treated animals compared with vehicle animals (Fig. 5a,b). The SD of membrane potential values in $30 \mathrm{sec}$ periods of recording obtained from haloperidoltreated rats was $3.6 \pm 0.06 \mathrm{mV}$, higher than the $1.8 \pm 0.03 \mathrm{mV}$ observed in vehicle animals ( $p<0.00001$; unpaired $t$ test). This is probably a result of an increase in the number of small spontaneous EPSPs in haloperidol-treated animals (Fig. 5b, inset).

VTA stimulation evoked prolonged membrane depolarizations with spike firing in most vehicle animals (Fig. $5 c, t o p)(n=3$ of 5 ; $60 \%$ ). Conversely, none of the haloperidol-treated animals exhibited increased spike firing during the VTA-evoked depolarization (Fig. $5 c$, bottom) $(n=0$ of $19 ; 0 \%)$, a response similar to what was observed in naive and sham animals (Fig. 4). Although the amplitude and decay to half amplitude in the VTA-evoked depolarization were not different (Fig. 5e,f), the proportion of cells showing spike firing in response to VTA stimulation was significantly lower in haloperidol-treated animals than in vehicle animals (Fig. $5 g)(p=0.0049$; Fisher's exact test). In addition, responses to PFC afferent activation were examined by singlepulse electrical stimulation $(1.0 \mathrm{~mA})$ of the PFC. Amplitudes of PFC-evoked EPSPs were significantly smaller in haloperidoltreated animals than in vehicle animals (Fig. $5 d, h)[7.5 \pm 2.8 \mathrm{mV}$ in haloperidol-treated animals $(n=12) ; 15.5 \pm 1.7 \mathrm{mV}$ in vehicle animals $(n=5)$; unpaired $t$ test; $p<0.00005$ ]. Latency and duration of EPSPs were not changed by drug treatment (Fig. 5i,j). These results indicate that haloperidol treatment prevents the appearance of abnormal firing in response to VTA stimulation and reduces the efficacy of corticoaccumbens synaptic activity.

\section{DISCUSSION}

Physiological properties of NAcc neurons and synaptic responses to cortical and DA afferent activation were studied in rats with a neonatal $\mathrm{VH}$ lesion. Although NAcc neurons did not show any alteration in response to PFC stimulation in rats with a neonatal VH lesion, their response to VTA stimulation was dramatically affected. VTA stimulation with trains of pulses mimicking DA cell burst firing evoked a membrane potential depolarization, as reported previously in intact animals (Yim and Mogenson, 1988; Goto and O'Donnell, 2001b), in all groups except in rats lesioned as adults. In normal rats and all control groups in this study, VTA stimulation reduced or suppressed spike firing. However, increased spike firing during VTA-evoked depolarization was observed in adult but not prepubertal rats with a neonatal $\mathrm{VH}$ lesion. Subchronic haloperidol treatment prevented the altered NAcc response to VTA stimulation. This indicates that a delayed alteration in the response of accumbens neurons to mesolimbic activation appears after a neonatal VH lesion, and that antipsychotic treatment restores the normal response.

When a VH lesion was performed in adult rats, transitions to the up state could not be observed. This is consistent with previous studies suggesting that up transitions depend on $\mathrm{VH}$ inputs to the NAcc (O'Donnell and Grace, 1995; Goto and
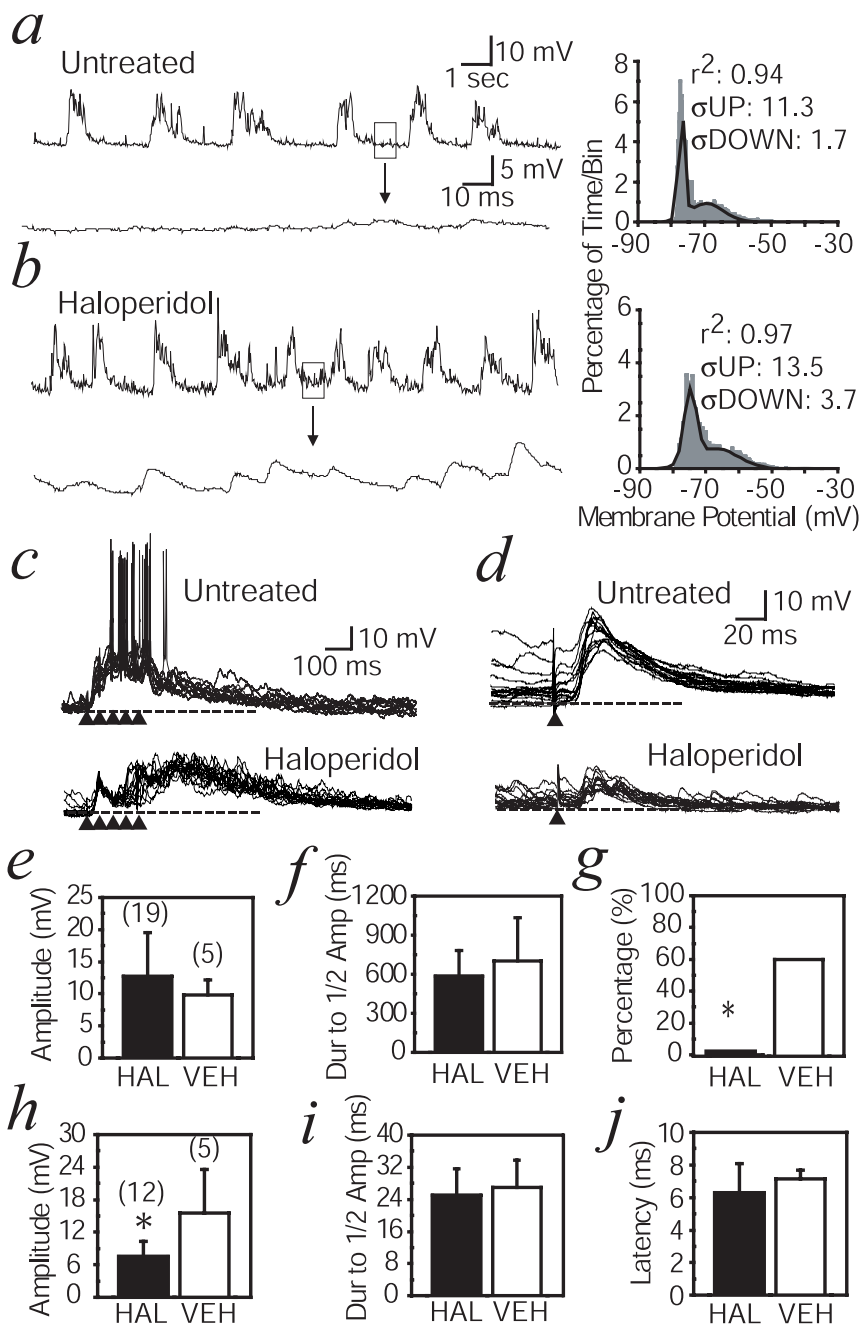

Figure 5. Effects of subchronic haloperidol treatment on NAcc neuron response to VTA and PFC activation. $a$, Representative trace of spontaneous membrane potential activity in an NAcc neuron from a neonatally lesioned rat. The bottom trace expands the boxed area during the down state at a faster time scale. The histogram on the right shows the membrane potential distribution from the traces shown on the left. The dark line is the dual Gaussian function that fits best to the distribution. The SDs $(\sigma)$ of up and down states are indicated. $b$, Similar representative trace of spontaneous membrane potential activity in an NAcc neuron from a neonatally lesioned rat with subchronic haloperidol treatment. The bottom trace expands the boxed down state area at a faster time scale, showing small spontaneous EPSPs. Larger $\sigma$ s in both up and down states are indicated in the histogram to the right. c, Overlays of responses to VTA stimulation in a neonatally lesioned rat (untreated; top) and a neonatally lesioned rat with subchronic haloperidol treatment (bottom). Arrowheads indicate trains of pulses. A depolarization is observed in both cases, but no spike firing could be evoked in animals treated with haloperidol, similar to what was observed in naive animals. $d$, Overlays of responses to PFC stimulation (arrowhead) in a neonatally lesioned rat (untreated; top) and a neonatally lesioned rat with subchronic haloperidol treatment (bottom). $e, f$, Bar graphs comparing amplitude and decay with half amplitude of VTA-evoked depolarizations. Numbers in parentheses indicate the number of recordings. $H A L$ and $V E H$ indicate haloperidoltreated and vehicle (water only) animals, respectively. Dur, duration; Amp, amplitude. g, Bar graph comparing number of neurons exhibiting spike firing in response to VTA stimulation, represented as a percentage. ${ }^{*} p=0.0049$ (Fisher's exact test). $h$, Bar graph comparing peak amplitude of EPSPs evoked by PFC stimulation. ${ }^{*} p<0.00005$, unpaired $t$ test. Numbers in parentheses indicate the number of recordings. $i, j$, Comparisons of PFC-evoked EPSP decay time with half of peak response and latency. Dashed lines in $c$ and $d$ indicate resting membrane potential. 
O’Donnell, 2001a). Surprisingly, VTA-evoked membrane depolarizations were also absent in adult-lesioned rats. One explanation would be that VTA stimulation evokes the transition to the up state by activation of hippocampal terminals. Indeed, there is evidence indicating that DA in the NAcc may enhance hippocampal terminal excitability (Yang and Mogenson, 1986), although there are also some data indicating a downregulation of hippocampal-NAcc responses by DA (Pennartz et al., 1992). Thus, VTA-evoked membrane potential depolarization may involve a control of hippocampal synaptic terminal efficacy within the NAcc, a mechanism that may involve DA and non-DA components of the mesolimbic system.

In rats with a neonatal VH lesion, step-like membrane potential fluctuations could still be detected. This indicates that there were enough synchronous glutamatergic inputs to drive NAcc neurons into the up state in these animals. Because the lesion took place early in development, the absence of normal $\mathrm{VH}$ afferents may have allowed effective connections with NAcc neurons from glutamatergic fibers that would have otherwise been eliminated. The absence of up states in animals with an adult $\mathrm{VH}$ lesion also indicates the developmental context of this effect. The increased regularity of up transitions in neonatal VH-lesioned rats suggests that such compensatory synaptic inputs may arise from a brain region with very regular, oscillatory electrical activity. Although speculative, the subthalamic nucleus (STN) may be considered as a candidate. The STN is known to project to the NAcc (Groenewegen and Berendse, 1990), although this projection is normally sparse. The presence of this increased regularity in prepubertal animals suggests that this phenomenon is independent of the changes yielding abnormal evoked responses to VTA stimulation. It can be speculated that a loss of $\mathrm{VH}$ inputs may result in increased synaptic projections from the STN or other brain regions to the NAcc.

NAcc neurons in rats with a neonatal $\mathrm{VH}$ lesion responded to VTA stimulation with increased rather than decreased spike firing. This was observed in adult but not prepubertal rats. A number of mechanisms could be advanced to explain this finding. First, increased spike firing by VTA stimulation could be caused by enhanced excitatory inputs to NAcc neurons. We have shown recently that VTA stimulation evokes prolonged membrane potential depolarizations accompanied by reduction in spike firing in PFC pyramidal neurons (Lewis and O'Donnell, 2000). However, in neonatally lesioned rats, PFC pyramidal neurons show a prolonged up state with increased spike firing in response to VTA stimulation (O'Donnell et al., 2002). Although the mechanisms resulting in such an increase are not clear, an enhanced VTAevoked cortical firing in neonatally lesioned rats could in turn evoke abnormal spike firing in the NAcc. A role of the PFC in the behavioral consequences of a neonatal $\mathrm{VH}$ lesion had been indicated by the ability of PFC lesions to improve abnormal behaviors in these animals (Lipska et al., 1998). An alternative mechanism could involve abnormalities of DA receptor expression in NAcc neurons as a consequence of the neonatal $\mathrm{VH}$ damage. For example, there is evidence of altered NAcc D3 DA receptor expression in these animals (Flores et al., 1996). In this regard, it is worth considering that the mesocortical innervation increases continuously until P60 (Kalsbeek et al., 1988). Thus, the absence of changes before puberty may be related to an absence of a mature DA projection. Regardless of the mechanism, the abnormal response of NAcc neurons to VTA activation can explain the exaggerated reaction to DA agonists and stress that can be observed in adult but not prepubertal rats with a neonatal VH lesion (Lipska et al., 1993, 1995).

The alterations reported here were independent of the extent of lesion. Animals with a lesion as small as that shown in Figure $1 a$ or as large as that shown in Figure $1 b$ exhibited increased firing in response to VTA stimulation. This may be related to the exquisite sensitivity of the hippocampal projection neurons during this critical developmental stage. Indeed, a recent study has shown that behavioral deficits similar to those elicited by this lesion are observed in animals with a reversible ventral hippocampal inactivation produced by tetrodotoxin injection at $\mathrm{P} 6-\mathrm{P} 7$ (Lipska et al., 2002). Thus, a transient disturbance in the VH may be what is behind the delayed and long-lasting effects of these lesions, independently of their size.

VTA stimulation did not result in altered NAcc activity in haloperidol-treated animals. It has been shown previously that subchronic antipsychotic treatment normalized abnormal behaviors in animals with a neonatal VH lesion (Lipska and Weinberger, 1994), suggesting that the increased spike firing to VTA activation observed in NAcc neurons may be related to the behavioral anomalies in adult animals with a neonatal $\mathrm{VH}$ lesion. The amplitude of PFC-evoked EPSPs was also reduced by haloperidol treatment. The increased NAcc firing in response to VTA stimulation appears to be dependent on increased PFC firing and may be necessary for the expression of behavioral deficits. Both PFC lesion (Lipska et al., 1998) and haloperidol treatment (Lipska and Weinberger, 1994) could improve behaviors by dampening the exaggerated PFC drive of NAcc neurons.

Mesocorticolimbic system alterations arising from a neurodevelopmental disturbance have been proposed as a pathophysiological mechanism for schizophrenia (Waddington, 1993; Weinberger and Lipska, 1995). Animals that develop with a disorganized hippocampus exhibit an altered PFC response to DA (O'Donnell et al., 2002). Because it has been shown that projections from the PFC to the VTA regulate DA cell spike firing (Tong et al., 1996), the increased PFC firing in response to VTA stimulation may result in an additional increase in VTA activity and DA release in the NAcc, bringing about the PFC dysfunction and DA hyperactivity that characterize psychosis (Laruelle, 2000). The subsequent enhancement of NAcc activity could provide a link between the DA-PFC dysfunction and schizophrenia symptoms. The pathophysiological changes in schizophrenia are still matter of debate. For example, deficits in GABA PFC transmission have been indicated (Lewis, 2000), but there is also evidence that these may be related to antipsychotic medication (Benes et al., 2000). Also, the changes in schizophrenia are observed primarily in the dorsolateral PFC, an area that does not exist in rodents. However, the medial PFC in rats controls DA cell activity, as is also the case for the primate dorsolateral PFC (Bertolino et al., 1999). Thus, although different, the medial PFC in rodents and dorsolateral PFC in primates share an important modulation that is relevant to our findings.

The ability of subchronic antipsychotic treatment to reverse both behavioral alterations and DA-PFC dysfunction strengthens the validity of the neonatal $\mathrm{VH}$ lesion as an animal model of schizophrenia. One should be aware, however, that this model has limitations. For example, there is no indication that schizophrenia patients exhibit actual hippocampal damage. This caveat may be minimized with the use of a transient inactivation of the hippocampus, as done recently by Lipska et al. (2002). Our findings provide experimental data indicating that delayed physiological 
alterations can occur in a brain system associated with schizophrenia as a consequence of neonatal hippocampal damage.

\section{REFERENCES}

Al-Amin HA, Weinberger DR, Lipska BK (2000) Exaggerated MK801 -induced motor hyperactivity in rats with the neonatal lesion of the ventral hippocampus. Behav Pharmacol 11:269-278.

Bachevalier J, Alvarado MC, Malkova L (1999a) Memory and socioemotional behavior in monkeys after hippocampal damage incurred in infancy or in adulthood. Biol Psychiatry 46:329-339.

Bachevalier J, Beauregard M, Alvarado MC (1999b) Long-term effects of neonatal damage to the hippocampal formation and amygdaloid complex on object discrimination and object recognition in rhesus monkeys (Macacca mulatta). Behav Neurosci 113:1127-1151.

Benes FM, Todtenkopf MS, Logotatos P, Williams M (2000) Glutamate decarboxylase(65)-immunoreactive terminals in cingulate and prefrontal cortices of schizophrenic and bipolar brains. J Chem Neuroanat 20:259-269.

Bertolino A, Knable MB, Saunders RC, Callicott JH, Kolachana B, Mattay VS, Bachevalier J, Frank JA, Egan M, Weinberger DR (1999) The relationship between dorsolateral prefrontal $N$-acetylaspartate measures and striatal dopamine activity in schizophrenia. Biol Psychiatry 45:660-667.

Caboche J, Vernier P, Rogard M, Julien JF, Mallet J, Besson MJ (1992) Role of dopaminergic D2 receptors in the regulation of glutamic acid decarboxylase messenger RNA in the striatum in the rat. Eur J Neurosci 4:438-447.

Chambers RA, Moore J, McEvoy JP, Levin ED (1996) Cognitive effects of neonatal hippocampal lesions in a rat model of schizophrenia. Neuropsychopharmacology 15:587-594.

Eagan KP, Partridge LD (1989) The sequential-interval state space: a means of displaying temporal information in neuron firing. J Neurosci Methods 27:245-252.

Feasey-Truger KJ, Earl CD, Alzheimer C, ten Bruggencate G (1995) Stimulus-evoked dopamine overflow in the rat nucleus accumbens is decreased following chronic haloperidol administration: an in vivo voltammetric study. Neurosci Lett 183:91-95.

Flores G, Barbeau D, Quirion R, Srivastava LK (1996) Decreased binding of dopamine D3 receptors in limbic subregions after neonatal bilateral lesion of rat hippocampus. J Neurosci 16:2020-2026.

Goto Y, O'Donnell P (2001a) Synchronous activity in the hippocampus and nucleus accumbens in vivo. J Neurosci 21:RC131:1-5.

Goto Y, O'Donnell P (2001b) Network synchrony in the nucleus accumbens in vivo. J Neurosci 21:4498-4504.

Grace AA (1992) The depolarization block hypothesis of neuroleptic action: implications for the etiology and treatment of schizophrenia. J Neural Transm Suppl 36:91-131.

Groenewegen HJ, Berendse HW (1990) Connections of the subthalamic nucleus with ventral striatopallidal parts of the basal ganglia in the rat. J Comp Neurol 294:607-622.

Groenewegen HJ, Vermeulen-Van der Zee E, te Kortschot A, Witter MP (1987) Organization of the projections from the subiculum to the ventral striatum in the rat: a study using anterograde transport of Phaseolus vulgaris leucoagglutinin. Neuroscience 23:103-120.

Kalsbeek A, Voorn P, Buijs RM, Pool CW, Uylings HB (1988) Development of the dopaminergic innervation in the prefrontal cortex of the rat. J Comp Neurol 269:58-72.

Kovelman JA, Scheibel AB (1984) A neurohistological correlate of schizophrenia. Biol Psychiatry 19:1601-1621.

Laruelle M (2000) The role of endogenous sensitization in the pathophysiology of schizophrenia: implications from recent brain imaging studies. Brain Res Brain Res Rev 31:371-384.

Lewis BL, O'Donnell P (2000) Ventral tegmental area afferents to the prefrontal cortex maintain membrane potential "up" states in pyramidal neurons via $\mathrm{D}_{1}$ dopamine receptors. Cereb Cortex 10:1168-1175.

Lewis DA (2000) GABAergic local circuit neurons and prefrontal cortical dysfunction in schizophrenia. Brain Res Brain Res Rev 31:270-276.

Lipska BK, Weinberger DR (1994) Subchronic treatment with haloperidol and clozapine in rats with neonatal excitotoxic hippocampal damage. Neuropsychopharmacology 10:199-205.

Lipska BK, Weinberger DR (2000) To model a psychiatric disorder in animals: schizophrenia as a reality test. Neuropsychopharmacology 23:223-239.

Lipska BK, Jaskiw GE, Weinberger DR (1993) Postpubertal emergence of hyperresponsiveness to stress and to amphetamine after neonatal excitotoxic hippocampal damage: a potential animal model of schizophrenia. Neuropsychopharmacology 9:67-75.

Lipska BK, Chrapusta SJ, Egan MF, Weinberger DR (1995) Neonatal excitotoxic ventral hippocampal damage alters dopamine response to mild repeated stress and to chronic haloperidol. Synapse 20:125-130.

Lipska BK, Swerdlow NR, Geyer MA, Jaskiw GE, Braff DL, Weinberger DR (1996) Neonatal excitotoxic hippocampal damage in rats causes post-pubertal changes in prepulse inhibition of startle and its disruption by apomorphine. Psychopharmacology (Berl) 122:35-43.

Lipska BK, Al-Amin HA, Weinberger DR (1998) Excitotoxic lesions of the rat medial prefrontal cortex: effects on abnormal behaviors associated with neonatal hippocampal damage. Neuropsychopharmacology 19:451-464.

Lipska BK, Halim ND, Segal PN, Weinberger DR (2002) Effects of reversible inactivation of the neonatal ventral hippocampus on behavior in the adult rat. J Neurosci 22:2835-2842.

Marcus MM, Nomikos GG, Malmerfelt A, Zachrisson O, Lindefors N, Svensson TH (1997) Effect of chronic antipsychotic drug treatment on preprosomatostatin and preprotachykinin A mRNA in the prefrontal cortex, the nucleus accumbens and the caudate-putamen of the rat. Brain Res Mol Brain Res 45:275-282.

O'Donnell P, Grace AA (1995) Synaptic interactions among excitatory afferents to nucleus accumbens neurons: hippocampal gating of prefrontal cortical input. J Neurosci 15:3622-3639.

O'Donnell P, Grace AA (1998) Dysfunctions in multiple interrelated systems as the neurobiological bases of schizophrenic symptom clusters. Schizophr Bull 24:267-283.

O'Donnell P, Greene J, Pabello N, Lewis BL, Grace AA (1999) Modulation of cell firing in the nucleus accumbens. Ann NY Acad Sci 877:157-175.

O’Donnell P, Lewis BL, Lipska BK, Weinberger DR (2002) Neonatal hippocampal damage alters electrophysiological properties of prefrontal cortical neurons in adult rats. Cereb Cortex 12:975-982.

Paxinos G, Watson C (1998) The rat brain in stereotaxic coordinates. San Diego, CA: Academic.

Pennartz CMA, Dolleman Van der Weel MJ, Kitai ST, Lopes da Silva FH (1992) Presynaptic dopamine D1 receptors attenuate excitatory and inhibitory limbic inputs to the shell region of the rat nucleus accumbens studied in vitro. J Neurophysiol 67:1325-1334.

Sams Dodd F, Lipska BK, Weinberger DR (1997) Neonatal lesions of the rat ventral hippocampus result in hyperlocomotion and deficits in social behaviour in adulthood. Psychopharmacology (Berl) 132:303-310.

Thierry AM, Blanc G, Sobel A, Stinus L, Glowinski J (1973) Dopaminergic terminals in the rat cortex. Science 182:499-501.

Tong ZY, Overton PG, Clark D (1996) Stimulation of the prefrontal cortex in the rat induces patterns of activity in midbrain dopaminergic neurons which resemble natural burst events. Synapse 22:195-208.

Volk DW, Austin MC, Pierri JN, Sampson AR, Lewis DA (2000) Decreased glutamic acid decarboxylase 67 messenger RNA expression in a subset of prefrontal cortical gamma-aminobutyric acid neurons in subjects with schizophrenia. Arch Gen Psychiatry 57:237-245.

Voorn P, Jorritsma Byham B, Van Dijk C, Buijs RM (1986) The dopaminergic innervation of the ventral striatum in the rat: a light- and electron-microscopical study with antibodies against dopamine. J Comp Neurol 251:84-99.

Waddington JL (1993) Schizophrenia: developmental neuroscience and pathobiology. Lancet 341:531-536.

Weinberger DR (1999) Cell biology of the hippocampal formation in schizophrenia. Biol Psychiatry 45:395-402.

Weinberger DR, Lipska BK (1995) Cortical maldevelopment, antipsychotic drugs, and schizophrenia: a search for common ground. Schizophr Res 16:87-110.

Yang CR, Mogenson GJ (1986) Dopamine enhances terminal excitability of hippocampal-accumbens neurons via D2 receptor: role of dopamine in presynaptic inhibition. J Neurosci 6:2470-2478.

Yim CY, Mogenson GJ (1988) Neuromodulatory action of dopamine in the nucleus accumbens: an in vivo intracellular study. Neuroscience 26:403-415. 